\title{
Comparing QD and OLED Electronic Displays Technology
}

\author{
Behzad Niazi Damaghi \\ Engineering Department, Iran Office Machines Industries (IOMIND) Company, \\ Alborz Province, Iran
}

\begin{abstract}
In this paper we will try to give a good and sufficient compare between two new technologies in providing electronic displays: one is Quantum Dot (QD) technology and the other one is Organic Light Emitting Diode (OLED) technology. Using of CIE 1931 color space standard helped us to show color gamut plots differences in different technologies. We would evaluate the LCD and LED positive and negative aspects and based on this analysis between different new technologies we can get useful opinions to optimizing the manufacturing of electronic displays.

Our compare includes below aspects:

1. Color Gamut

2. System efficiency

3. Manufacturing procedure

4. Cost efficiency

5. Shelf life

6. Impact on environment

7. Contrast Ratio

8. Response Time

Also we measured these items under the same conditions as temperature, humidity, ambient light and other environmental conditions. At the end we made a conclusion to choose the better technology by considering tradeoff between all important aspects. In some parts we had to discuss about conventional technologies like other types of LCDS.
\end{abstract}

Keywords: QD, OLED, Colour Gamut, Shelf life, Contrast Ratio, Response Time, CIE 1931 colour space.

\section{Introduction}

Welcome to 6th International Conference on Computer, Electrical and Electronics Engineering \& Technology (ICEEET2017).

Nowadays, our daily lives have a strong connection with electronic displays as you can see them in many electronic devices and systems. The main part of the electronic displays belongs to LCDs. LCDs have a large variety types. Besides LCDs a new comer technology is growing rapidly and it is OLED technology. In this article we want to compare these new technologies with each other. We know LCDs are very ubiquitous now but it is remarkable to consider this fact that OLED technology has reached a good and satisfied stage among consumers of electronic displays. Conventional LCDs due to low color gamut percent and low system efficiency against new technologies were being put aside easily for example Cold cathode fluorescent lamp (CCFL) was once the most prevalent backlight source, but its color gamut is only $\sim 75 \%$ of NTSC (National Television Standard Committee) standard. In order to achieve wider color gamut, higher brightness, and lower power consumption, mercury-free white LED (WLED) has rapidly replaced CCFL as the major backlight source. Highquality white color emission is also an important aspect of liquid-crystal displays (LCD). However, conventional LCDs are known to reproduce less than $50 \%$ of the color that the human eye can see due to their relatively poor R-G-B light spectrum. Display manufacturers have developed a variety of new technologies, such as discrete 
RGB LED backlights and LEDs enhanced with yttrium aluminum garnet (YAG) with red phosphor. However, they still have issues with regard to color quality, scalability, cost and stability. Great effort has been made toward improving LCD color performance, as todays could be seen LCDs with LED backlight are yet serious rival for OLED, especially after introducing new technology which was named QLED. As a benefit of QLED we can mention larger color gamut than conventional LCDs with higher system efficiency also. In our paper first of all we try to give a brief explain and definition of LCDs, QLED and OLED technology and in the next step we would discuss about some electronic display aspects like color gamut, system efficiency, contrast ratio, response time, view angle,... in both new technologies and compare them to get a conclusion in order to the better choose for applying in various applications. This compare is useful for every organization and institute working on electronic displays to choose best choice regarding its purpose. In some parts for simplicity we will ignore discussing about some extra details but we try to present major points completely. [1]

\section{Definitions}

\subsection{LCD}

LCD stands for Liquid Crystal Display; it means by using Liquid Crystal we can produce our images to be shown on the electronic displays. As a general definition for LCD Display we can explain it as below:

LCD (liquid crystal display) is the technology used for displays in notebook, other smaller computers, in TVs and also smartphones. Like light-emitting diode (LED) and gas-plasma technologies, LCDs allow displays to be much thinner than cathode ray tube (CRT) technology. LCDs consume much less power than gas-display displays because they work on the principle of blocking light rather than emitting it. An LCD is made with either a passive matrix or an active matrix display grid. The active matrix LCD is also known as a thin film transistor (TFT) display because in this LCD type, pixels are formed by use of thin film transistors and pixel electrodes. The passive matrix LCD has a grid of conductors with pixels located at each intersection in the grid. A current is sent across two conductors on the grid to control the light for any pixel. An active matrix has a transistor located at each pixel intersection, requiring less current to control the luminance of a pixel. For this reason, the current in an active matrix display can be switched on and off more frequently; improving the screen refresh time (your mouse will appear to move more smoothly across the screen, for example). Some passive matrix LCD's have dual scanning, meaning that they scan the grid twice with current in the same time that it took for one scan in the original technology. However, active matrix is still a superior technology. The LCD works on the principle of blocking light. LCD TVs all work in the same way. At the back of the set is a backlight, which is on all of the time, producing the light that you see. In front of the backlight are two polarizing filters. The first blocks all vertical light waves, the second, rotated 90 degrees, blocks all horizontal light waves. Together, then, they block all the light from the backlight, creating a completely blank screen. That's no use, obviously, but this is where the layer of liquid crystals come in. in their natural state, a liquid crystal is twisted, changing the orientation of the light. This means that light is allowed to pass through the second filter, creating a completely white screen. To control the light through the filter, a voltage is applied to each liquid crystal. Controlling the voltage controls the twist, and therefore controls how much light makes it through the second filter. Using this system and one liquid crystal per pixel, you can create a black and white picture with shades of grey. In order to produce a fullcolour image, each pixel is actually made up of three sub-pixels. These sit, respectively, behind a colour filter of red, green and blue: controlling the light that passes through each of these sub-pixels allows the TV to produce a picture with thousands of colours. The big downside with this technology is that the filters aren't capable of completely blocking all light, so with a permanent backlight there's always a bit of light bleed and, depending on the quality of the set, blacks can look more like dark grey. There are two kinds of LCD panel in use today, the first is called "Twisted Nematic" or TN, and it requires voltage from an electrode above and below the LCD panel. The second technology is called "in plane switching" and instead uses two electrodes on one side of the panel. IPS panels are very common now, because they have far better viewing angles and more faithful colour reproduction. TN panels are used a lot, but generally in cheaper displays. They are still common in computer monitors, especially those aimed at gamers who value low response times over image quality. [2-5] 


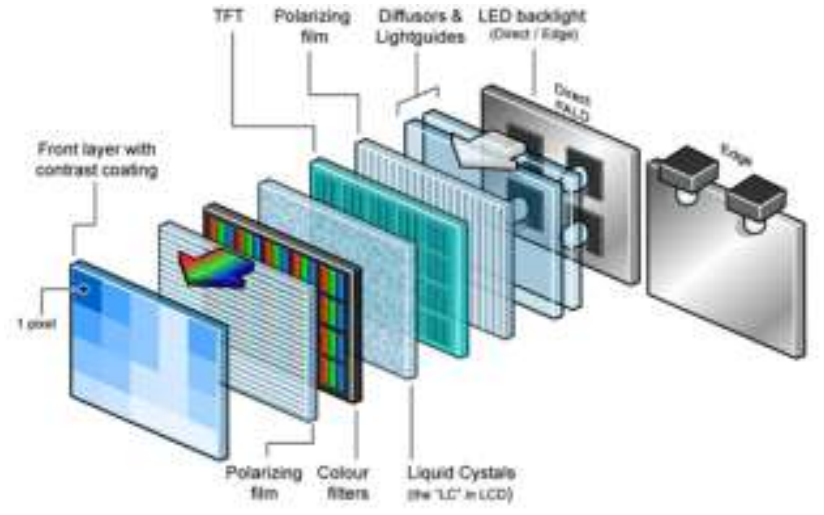

Fig. 1: Exploded View of TFT LCD.
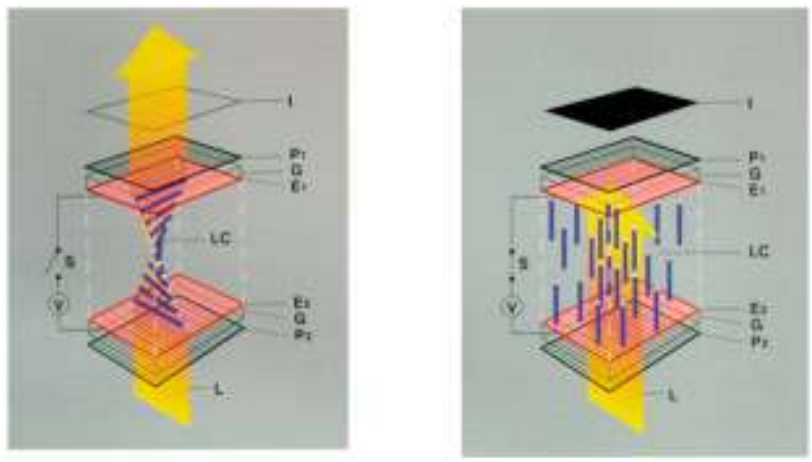

Fig. 2: How Liquid Crystal molecules change the light direction.

\subsection{OLED}

OLED stands for Organic Light Emitting Diode; each pixel in an OLED display is made of a material that glows when you jab it with electricity. Kind of like the heating elements in a toaster, but with less heat and better resolution. This effect is called electroluminescence, which is one of those delightful words that is big, but actually makes sense: "electro" for electricity, "lumin" for light and "escence" for, well, basically "essence". What's the "organic" part? The specific electroluminescent materials used in OLED displays are compounds, which mean they contain carbon plus some other ingredients. Each colour requires a different organic compound (though these aren't necessarily the colours you see on the TV, but more on that later).The effect is that each tiny OLED pixel in the screen creates light depending on how much electric current you send it. By increasing current, light increases. No current, no light, and that's one key to OLED's excellent picture quality. OLED TV marketing often claims "infinite" contrast ratios, and while that might sound like typical hyperbole, it's one of the extremely rare instances where such claims are actually true. Since OLED can produce a perfect black, emitting no light whatsoever, its contrast ratio (expressed as the brightest white divided by the darkest black) is technically infinite. And contrast ratio is arguably the most important aspect of picture quality. [6], [9]

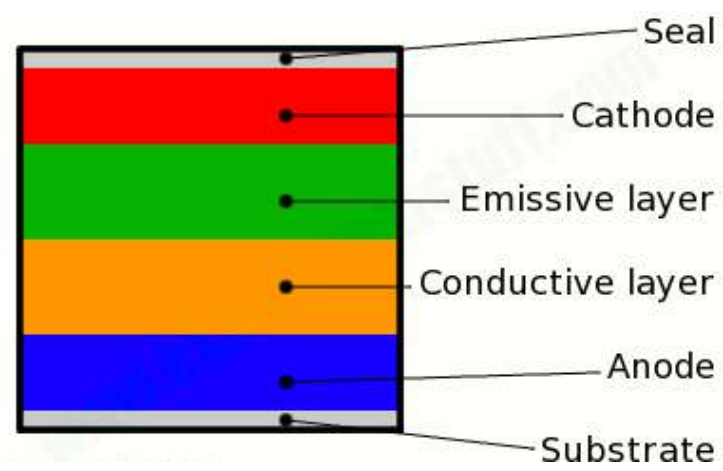

Fig. 3: Technology of OLED.

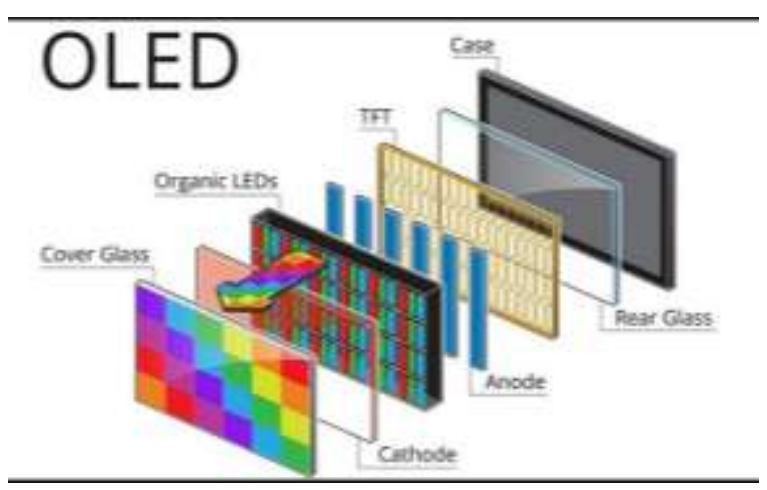

Fig. 4: Exploded View of OLED Display.

\subsection{QD Display and QLED Display}

\subsubsection{QD Display}

Quantum dots (QD) are very small semiconductor particles, only several nanometres in size, so small that their optical and electronic properties differ from those of larger particles. They are a central theme in nanotechnology. Many types of quantum dot will emit light of specific frequencies if electricity or light is applied to them, and these frequencies can be precisely tuned by changing the dots' size, shape and material, 
giving rise to many applications. In the language of materials science, Nano scale semiconductor materials tightly confine either electrons or electron holes. Quantum dots are also sometimes referred to as artificial atoms, a term that emphasizes that a quantum dot is a single object with bound, discrete electronic states, as is the case with naturally occurring atoms or molecules. Quantum dots exhibit properties that are intermediate between those of bulk semiconductors and those of discrete molecules. Their optoelectronic properties change as a function of both size and shape. Larger QDs (radius of 5-6 nm, for example) emit longer wavelengths resulting in emission colours such as orange or red. Smaller QDs (radius of 2-3 nm, for example) emit shorter wavelengths resulting in colours like blue and green, although the specific colours and sizes vary depending on the exact composition of the QD. Quantum dot (QD) technology is emerging as a novel back- light source that could help to increase the colour performance of LCDs. QDs are composed of nanoparticles with diameters of 5-20nm. When charge carriers (electrons and holes) are confined to such small particles, the larger band gap of the surrounding material creates a barrier. The discrete energy levels that arise as a result of this barrier consequently dictate the material properties. This effect (size quantization) gives QDs several attractive features, including high quantum efficiency, a broad absorption band, narrow emission line width, and a controllable emission peak. Figure 5 shows the emission properties of the QD samples that we used, which have a cadmium sulphide/selenide core and a zinc sulphide shell ( $\mathrm{CdSx}$ Se1-x/ZnS: the ratio of $\mathrm{CdS}$ is a variable, $\mathrm{x}$, while the ratio of $\mathrm{CdSe}$ is $1-\mathrm{x}$ ). Their luminescence is quite narrow (full width at half-maximum is $30 \mathrm{~nm}$ ), which yields highly saturated colour emissions. In addition to this, the emission wavelength can be tuned by varying both the size and composition of the QD during material synthesis. Cadmium Selenide QDs of different sizes, for example, can emit light from blue (450nm) through to red (700nm) wavelengths. So adding a Quantum Dot Layer to the LCD layers and changing the back light LED to a blue LED can make an LCD to QD display as you can see in Figure 6. [1], [7], [8], [17].

Quantum Dot Size and Color

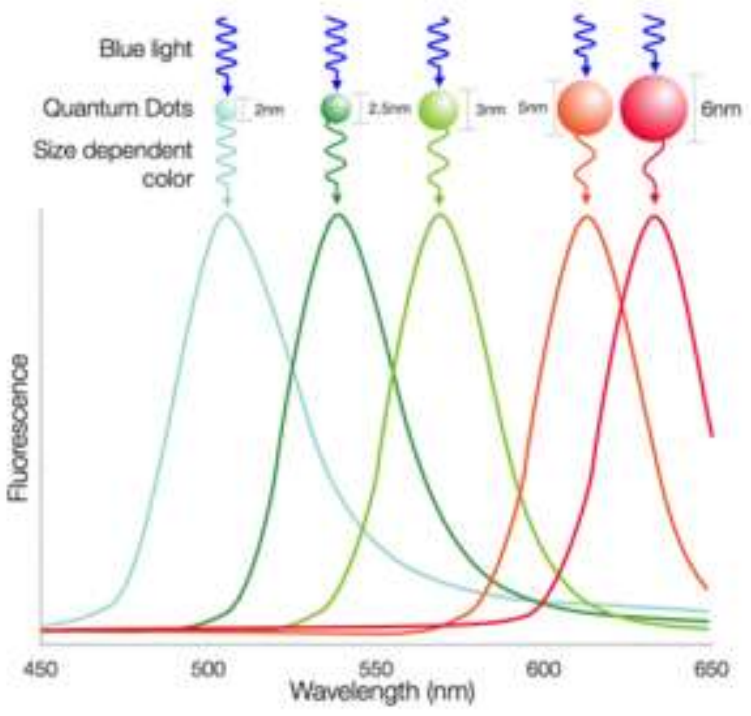

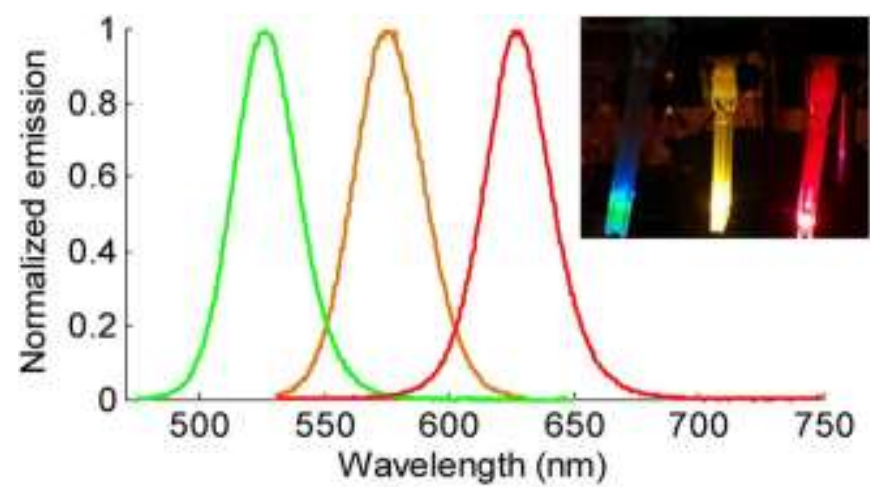

Wavelength $(\mathrm{nm})$

Fig. 5: Emission spectrum of a quantum dot (QD) with a cadmium sulphide/selenide core and a zinc sulphide shell (CdSx $\mathrm{Se} 1-\mathrm{x} / \mathrm{ZnS}$ : the ratio of $\mathrm{CdS}$ is $\mathrm{x}$, while the ratio of CdSe is $1-\mathrm{x})$. Varying the composition or particle size alters the band gap of the core, enabling a tuneable emission wavelength. Inset shows the emission colour when QDs are excited by a 459nm blue LED. 


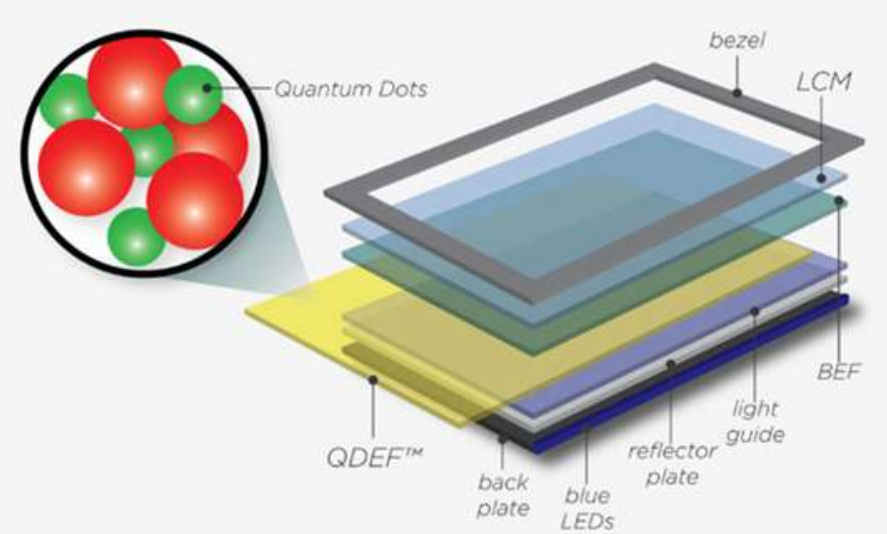

Fig. 6: Exploded view of QD display. QDEF layer is added to conventional LCDs and the back light is changed to Blue LED.

\subsubsection{QLED Display}

As a new and recent technology QLED changes the display world and solve the QD display issues. In this new technology rather than requiring a separate backlight for illumination, a QLED TV natively controls the light emitted by individual pixel like an OLED TV. QLED, therefore, theoretically combines the best of quantum dot and OLED technology - the clarity and deep blacks of OLED, the superior brightness and colour gamut of quantum dots. [7], [8]

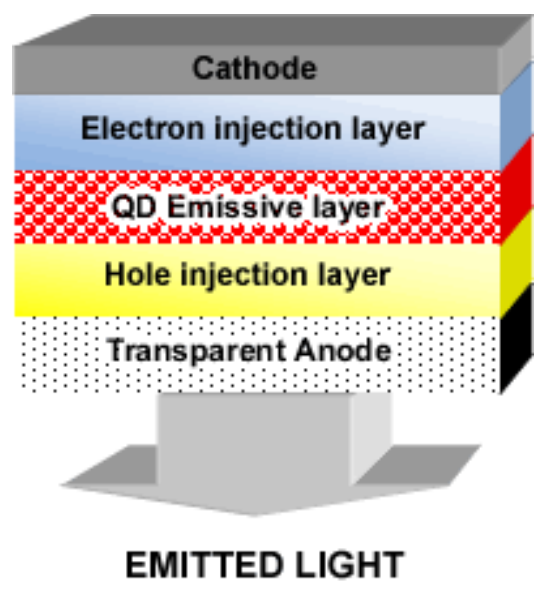

Fig. 7: QLED technology.

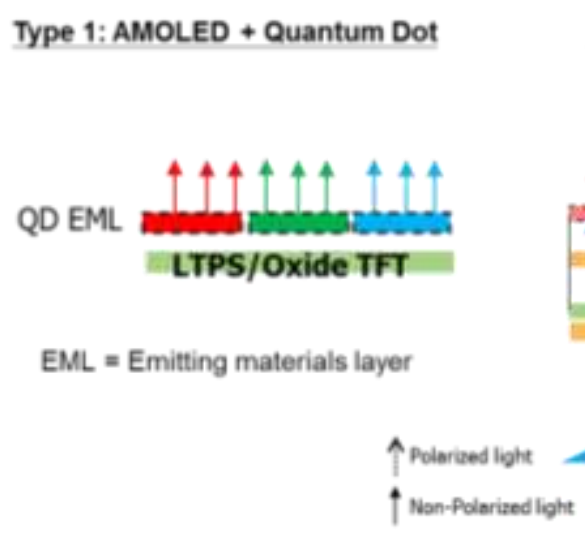

Type 2: Advanced LCD + Quantum Dot

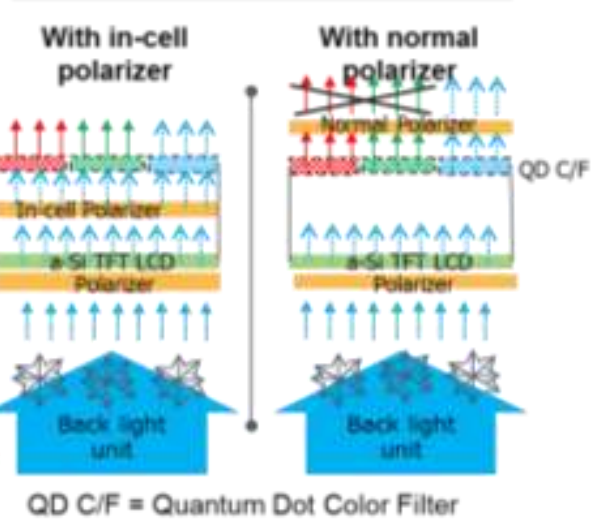

Fig. 8: QLED technology \& QD display technology.

\section{Compare}

\subsection{Color Gamut}

The entire range of colours available on a particular device determines colour gamut. The colour producing crystals are organic or inorganic is the primary difference. As we know, many of the first OLED had blue phosphors that only lasted about 7000 hours and we suspect the red and green phosphors were not much better. Now, those numbers have increased considerably. But OLEDs are made from rare earth materials and thus are expensive to produce as those materials become harder to get. QDs are made from inorganic semiconductor crystal substance which is one of the reason manufacturers are probably excited about the technology. It will likely be much less expensive to produce. Both OLED and QDEF are able to reproduce greater than 100 percentage of the NTSC colour gamut. As could be seen in Fig.9, QDEF can deliver more, especially in terms of red. This means that QDEF can offer more coverage, not just area, of more of the industry standard high gamut formats like Adobe RGB, DCI-P3 and NTSC. [1], [10], [16] 


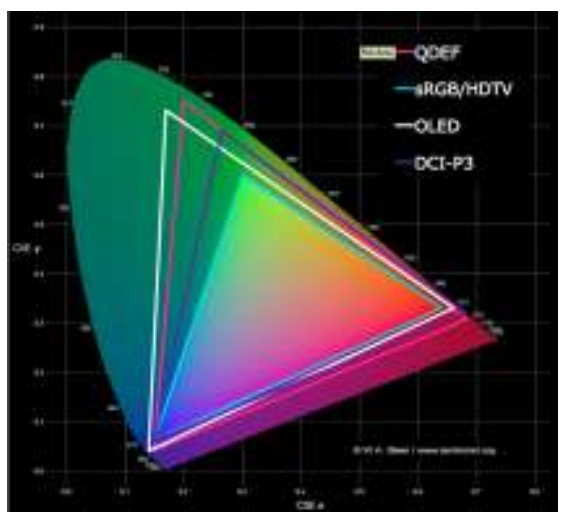

Fig. 9: Color Gamut Plot Of QDEF, OLED And HDTV.

TABLE 1: The Measured Color Coordinates (CIE1931) Values of OLED and QD Display

\begin{tabular}{|l|l|l|l|l|}
\hline Color Coordinates(CIE 1931) & RED & GREEN & BLUE & WHITE \\
\hline \multirow{3}{*}{ OLED } & $\begin{array}{l}\mathrm{Rx}=0.678 \\
\mathrm{Ry}=0.321\end{array}$ & $\begin{array}{l}\mathrm{Gx}=0.185 \\
\mathrm{~Gy}=0.738\end{array}$ & $\begin{array}{c}\mathrm{Bx}=0.142 \\
\mathrm{By}=0.050\end{array}$ & $\mathrm{~W} \mathrm{~W}=0.281$ \\
& & & & \\
\hline QD Display & $\mathrm{Rx}=0.288$ \\
& $\mathrm{Ry}=0.306$ & $\mathrm{Gx}=0.201$ & $\mathrm{Bx}=0.145$ & $\mathrm{Wx}=0.311$ \\
& & & $\mathrm{By}=0.048$ & $\mathrm{Wy}=0.320$ \\
\hline
\end{tabular}

\subsection{System Efficiency}

This is a difficult comparison to make since LCD and OLED are such different technologies. OLED is emissive, meaning each pixel is also a light source, and so the amount of power it consumes is dependent on content. For example, if a pixel is black, it is consuming no power while a white pixel can consume a significant amount of power due to the inefficiencies in the underlying material system, particularly in terms of blue, which are only 4-6 percentages efficient. This "zero power consumption for black" is a really nice story, but in practice, it's not very useful since we don't spend a lot of time looking at black screens. Screens with white backgrounds, like most of the web (i.e., Google), or e-book reading are much more challenging for OLED. QDEF is an LCD technology, so it relies on a backlight that stays on all the time. This sounds less efficient at first glance but QDEF is able to improve the efficiency of the system by tuning the spectrum of the light in the backlight to match the colour filters. This means less wasted light because you are only generating light that you will see and much more colour for the same amount of power input. So as a conclusion we can say OLED is more efficient unless QDEF tunes the spectrum of the light in the backlight. [9], [11], [14], [15], [16]

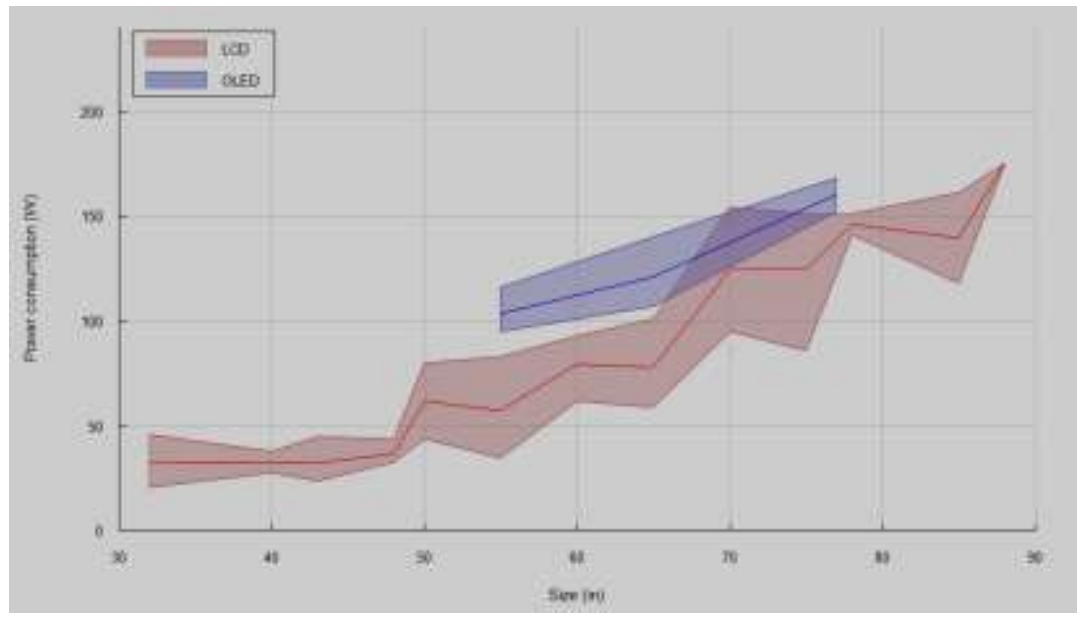

Fig. 10: Power consumption diagram for OLED and LCD technology 


\subsection{Manufacturing Procedure}

Due to this fact that OLEDs made from organic materials so the manufacturing procedure is a bit complex and hard because of high sensitivity of organic materials against moisture and oxygen so the manufacturing procedure is harder. Especially in large sizes the manufacturing procedure become more complicated than similar technologies like QD display and QLED. [9], [11]

\subsection{Cost Efficiency}

Because of using organic materials the cost of manufacturing is more than other technologies but nowadays by improving the organic materials production procedures the costs become more logical. On the other hand QD Display doesn't make a high increase in cost of a conventional LCD and as everyone knows LCDs are more cost efficient against OLEDs. [1], [9], [11]

\subsection{Reliability and Lifespan}

Nanosys spent a significant amount of time designing and testing QDEF to ensure it is of the highest quality and reliability. More than 100 patents have gone into the design of QDEF, and it has been tested to meet industry standard lifetimes for TV's (i.e., 50,000 hours or more). OLED has historically been limited by the performance of its blue component and the mismatch between red, green and blue lifetimes can lead to issues over the life of the display. As OLED manufacturers claim first OLEDs have 36,000 hours lifetimes but as the recent claim of OLED manufacture this lifetime has increased to 100,000 hours like LCDs. If the manufacturer claim is correct we can say there is no difference between OLEDs and QD Display lifespan. Also QLED is the same as QD display in the lifespan matter. [9], [11]

\subsection{Impact on Environment}

OLEDs are made of earth materials and this is not a good source to provide them because it is limited while the QD display made from inorganic materials and has no limit. [9], [11]

\subsection{Contrast Ratio and Black Level}

OLED wins here because of its ability to turn off individual pixels completely. It can produce truly perfect black. The better LCDs have local dimming, where parts of the screen can dim independently of others. This isn't quite as good as per pixel control because the black areas still aren't absolutely black, but it's better than nothing. The best LCDs have full array local dimming, which provides even finer control over the contrast of what's onscreen, but even they can suffer from "blooming," where a bright area spoils the black of an adjacent dark area. Contrast ratio is the difference between the brightest and the darkest a TV can be. OLED is the winner here because it can get extremely bright, plus it can produce absolute black with no blooming. It has the best contrast ratio of any modern display. Contrast Ratio of an OLED achieves more than 120,000 but for QD display and conventional LCDs is about 5000. [9], [11], [13]

\subsection{Response Time}

LCD panels take more time to switch from one color to another, and some are faster than others. The time it takes for a pixel to change state causes a trail to follow the moving object. Some TVs have worse decay than others, and when the response time is especially bad, an action scene can turn into a blurry mess. OLED TVs do not have this problem. Each of the pixels can change state virtually instantly, so no trail is left behind. For this reason, we consider OLED TVs to have no motion blur. Response time of an OLED TV in Grey to Grey colour transition is about 1ms and for QD display is about 8ms. [9], [11], [12]

\section{Conclusion}

Both new technologies try to increase image quality and purity of color but in some items OLED has been winner like response time, contrast ratio and view angle but in other items QD display is a good choice also as a newcomer technology QLED is the most efficient one because this technology tries to solve OLED and QD 
Display defects and incompetence it is the same as OLEDs with a main difference in using of inorganic (Quantum Dots) materials instead of organic materials.

\section{References}

[1] Wide color gamut LCD with a quantum dot backlight, Zhenyue Luo, Yuan Chen, and Shin-Tson Wu* CREOL, The College of Optics and Photonics, University of Central Florida, Orlando, Florida 32816, USA

[2] How LCD TV works, http://www.expertreviews.co.uk/tvs-entertainment/8075/how-lcd-tv-works

[3] Sakae Someya, Ryuuzoh Nashimoto, Hirofumi Suzuki, Katsuhiko Yarita, Shinji Matsumoto, Akira Sasano, Hideaki Taniguchi, Ryouji Oritsuki ;TFT active matrix liquid crystal display devices, Publication Number :US 5132820 A

[4] http://whatis.techtarget.com/definition/LCD-liquid-crystal-display

[5] Seigo Togashi, Liquid crystal display panel, Publication Number : US4345249 A

[6] https://www.cnet.com/news/what-is-oled-and-what-can-it-do-for-your-tv/

[7] Zhenyue Luo, Yuan Chen, and Shin-Tson Wu , Quantum dots: a new era for liquid crystal display backlight, 10.1117/2.1201402.005314, SPIE Newsroom

[8] What is QLED? Demystifying the future of TV tech , http://www.trustedreviews.com/opinion/what-is-qled-the-futureof-tv-tech-explained-2945941

[9] QD TV: What is it? Quantum Dot vs. OLED TV,http://oled.lcdtvbuyingguide.com/oled-tv-articles/quantum-dottv.html ; LED LCD vs. OLED: TV display technologies compared, https://www.cnet.com/how-to/led-lcd-vs-oled/

[10] PC Magazine Encyclopedia , https://www.pcmag.com/encyclopedia/term/39985/color-gamut

[11] OLED and LED TV Power Consumption and Electricity Cost , http://www.rtings.com/tv/learn/led-oled-powerconsumption-and-electricity-cost

[12] QLED vs OLED vs LED TV: Which one is the best? , http://www.rtings.com/tv/reviews/by-type/qled-vs-oled-vs-led

[13] LED LCD vs. OLED: TV display technologies compared , https://www.charlesmilander.com/news/2017/04/led-lcd-vsoled-tv-display-technologies-compared-cnet/

[14] Science « Great Things from Small Things .. Nanotechnology, https://genesisnanotech.wordpress.com/tag/science/page/41/?iframe=true\&preview=true $\% 2 \mathrm{Ffeed} \% 2 \mathrm{~F}$

[15]QDEF - A Stepping Stone or Alternative to OLED? | HomeToys, https://www.hometoys.com/content.php?post_type=1531

[16]QDEF - A Stepping Stone or Alternative to OLED? - iconnect.ie, http://www.iconnect.ie/92-news/340-qdef-a-steppingstone-or-alternative-to-oled.html

[17] Quantum dot - Wikipedia, https://en.wikipedia.org/wiki/Quantum_dot 